\title{
Overview of Theory and Modeling in the Heavy Ion Fusion Virtual National Laboratory
}

R. C. Davidson, I. D. Kaganovich, W. W. Lee, H. Qin, E. A. Startsev, and S. Tzenov Plasma Physics Laboratory, Princeton University, Princeton, NJ 08543

A. Friedman, J. J. Barnard, R. H. Cohen, D. P. Grote, S. M. Lund, and W. M. Sharp Lawrence Livermore National Laboratory, University of California, Livermore, CA 94550

C. M. Celata, M. de Hoon, E. Henestroza, E. P. Lee, S. S. Yu, and J.-L. Vay Lawrence Berkeley National Laboratory, University of California, Berkeley, CA 94720

D. R. Welch and D. V. Rose

Mission Research Corporation, Albuquerque, NM 87110

C. L. Olson

Sandia National Laboratories, Albuquerque, NM 87110

(Dated: May 8, 2002) 


\begin{abstract}
This paper presents analytical and simulation studies of intense heavy ion beam propagation, including the injection, acceleration, transport and compression phases, and beam transport and focusing in background plasma in the target chamber. Analytical theory and simulations that support the High Current Experiment (HCX), the Neutralized Transport Experiment (NTX), and the advanced injector development program, are being used to provide a basic understanding of the nonlinear beam dynamics and collective processes, and to develop design concepts for the next-step Integrated Beam Experiment (IBX), an Integrated Research Experiment (IRE), and a heavy ion fusion driver. 3-D nonlinear perturbative simulations have been applied to collective instabilities driven by beam temperature anisotropy, and to two-stream interactions between the beam ions and any unwanted background electrons; 3-D particle-in-cell simulations of the $2 \mathrm{MV}$ Electrostatic Quadrupole (ESQ) injector have clarified the influence of pulse rise time; analytical studies and simulations of the drift compression process have been carried out; syntheses of a 4-D particle distribution function from phase-space projections have been developed; and studies of the generation and trapping of stray electrons in the beam self fields have been performed. Particlein-cell simulations, involving pre-formed plasma, are being used to study the influence of charge and current neutralization on the focusing of the ion beam in NTX and in a fusion chamber.
\end{abstract}




\section{INTRODUCTION}

This paper summarizes recent analytical and simulation studies of intense ion beam propagation carried out under the auspices of the Heavy Ion Fusion Virtual National Laboratory, with the goal of a detailed understanding of the intense beams which will be needed for an Inertial Fusion Energy driver. This paper is organized according to: basic studies of nonlinear beam dynamics and collective processes (Sec. II); studies in support of ongoing driver-related experiments, in particular the High-Current Experiment (HCX) at Lawrence Berkeley National Laboratory (LBNL) and the injector experiments at Lawrence Livermore National Laboratory (LLNL) (Sec. III); simulations of beam propagation in preformed plasma, relevant to both the Neutralized Transport Experiment (NTX) beginning at LBNL and the fusion chamber of a power plant (Sec. IV); and studies aimed at developing future experiments, especially an Integrated Beam Experiment (IBX) and an Integrated Research Experiment (IRE), and the simulation tools to model them (Sec. V).

\section{NONLINEAR BEAM DYNAMICS AND COLLECTIVE PROCESSES}

Considerable theoretical progress in the understanding of nonlinear beam dynamics has been made in several areas since the 2000 Heavy Ion Fusion Symposium[1]. Examples include: detailed analytical and nonlinear perturbative simulation studies of collective processes, including the electron-ion two-stream instability[2-7], and the Harrislike temperature-anisotropy instability driven by $T_{\perp b} \gg T_{\| b}[8-11]$; development of a selfconsistent theoretical model of charge and current neutralization for intense beam propagation through background plasma in the target chamber[12-15]; development of a robust theoretical model of beam compression dynamics and nonlinear beam dynamics in the final focus system using a warm-fluid description[16]; development of an improved kinetic description of nonlinear beam dynamics using the Vlasov-Maxwell equations[2, 17-20], including identification of the class of (stable) beam distributions, and the development of Hamiltonian averaging techniques that clarify the smooth-focusing approximation; the determination of a nonlinear bound on emittance growth due to the collective relaxation of space-charge nonuniformities [21]; a precise solution of the coupled envelope equations with

space charge [22]; and the role of transverse mismatch effects in final pulse compression 
$[23,24]$, to mention a few examples. We summarize below several key theoretical results obtained in these studies.

3D Multispecies Nonlinear Perturbative Simulation Studies of the Two-Stream Instability: A three-dimensional nonlinear perturbative $(\delta f)$ particle simulation scheme has been developed for studying the stability and transport properties of intense charged particle beams $[3,4]$. As a numerical method to solve the nonlinear Vlasov-Maxwell equations, the scheme splits the distribution function into equilibrium and perturbed parts[3, 4]. The perturbed distribution function is represented as a weighted summation over discrete particles, where the particle orbits are advanced by equations of motion in the focusing field and self-consistent fields, and the particle weights $w_{j}$ are advanced by the coupling between the perturbed fields and the zero-order distribution function $f_{j}^{0}$. The nonlinear $\delta f$ scheme exhibits minimal noise and accuracy problems in comparison with standard particlein-cell simulations. This simulation scheme is implemented in the newly developed Beam Equilibrium Stability and Transport (BEST) code[2-4]. This code provides an effective numerical tool to investigate collective instabilities, periodically-focused beam propagation in alternating-gradient focusing fields, halo formation, and any other important nonlinear processes in intense beam propagation. In the absence of background electrons, the BEST code has been used to demonstrate quiescent (stable) beam propagation over thousands of equivalent lattice periods[2]. In the presence of background electrons, however, a strong two-stream instability is observed[3,4], leading to a dipole $(m=1)$ transverse displacement of the beam ions and background electrons, which has been benchmarked against measurements of the electron-proton (e-p) two-stream instability in the Proton Storage Ring (PSR) experiment. The BEST code provides an important tool in the theoretical studies. For example, the nonlinear $\delta f$-simulation scheme has also been applied to the case of a periodic-focusing solenoidal field configuration, and quiescent, matched-beam propagation of a thermal equilibrium beam has been demonstrated at high beam intensities over hundreds of lattice periods[26], and detailed properties of the two-stream instability have been determined as a function of beam intensity and axial momentum spread $[3,4,6]$.

Collective Instability Driven by Large Temperature Anisotropy $\left(T_{\perp b} \gg T_{\| b}\right)$ : While considerable progress has been made in analytical investigations based on the Vlasov-Maxwell equations, such kinetic analyses are often complex, even under idealized assumptions. It is therefore important to develop and test the robustness of theoretical models to describe prop- 
agation of space-charge-dominated (low-emittance) beams in periodic-focusing transport systems, and to describe collective stability properties of high-intensity beams. In beams with strongly anisotropic distributions $\left(T_{\| b} / T_{\perp b} \ll 1\right)$ it is well known that a Harris-like collective instability[11] may develop[8-10] if there is sufficient coupling between the transverse and longitudinal degrees of freedom. Such anisotropies develop naturally in accelerators and may lead to a deterioration of beam quality. Both kinetic and fluid descriptions predict instability. The 3D nonlinear perturbative particle simulation code BEST has been used[8] to study the stability properties of intense nonneutral charged particle beams with strong temperature anisotropy. The most unstable modes have been identified and their eigenfrequencies and radial mode structure have been determined for axisymmetric perturbations[8]. Simulation results show that moderately intense beams with $s_{b}=\omega_{p b}^{2} / 2 \gamma_{b}^{2} \omega_{\beta \perp}^{2} \gtrsim 0.5$ are linearly unstable to short-wavelength perturbations with $k_{z}^{2} r_{b}^{2} \gtrsim 1$, provided the ratio of longitudinal to transverse temperatures is smaller than some threshold value. In the nonlinear saturation stage, the total distribution function is still far from equipartitioned, and free energy is available to drive an instability of the hydrodynamic type. In future simulations, we will carry out a systematic analysis of this instability using the 3D nonlinear $\delta f$ simulation code BEST to determine the instability's dependence on beam intensity, its nonlinear saturation properties, and the effects of the instability on beam emittance.

Self-Consistent Model of Ion Beam Charge and Current Neutralization by Background Plasma: The propagation of a high-current finite-length ion charge bunch through a background plasma is of interest for many applications[12-15], including heavy ion fusion. Models for charge and current neutralization have been studied both analytically and numerically during ion beam entry, propagation, and exit from the plasma. A suite of codes has been developed for calculating the degree of charge and current neutralization of the ion beam pulse by the background plasma[12]. The code suite consists of two different codes: a fully electromagnetic, relativistic particle-in-cell (PIC) code, and a relativistic Darwin model for long charge bunches. As a result of a number of simplifications, the second code is hundreds of times faster than the first one and can be used for most cases of practical interest, while the first code provides important benchmarking for the second. The analytical theory has been derived using the assumption of long charge bunches and conservation of generalized vorticity. The analytical results agree well with the results of numerical simulations for ion beam charge and current neutralization[12]. The numerical simulations also show complex 
phenomena, including collective wave excitations, during beam entry into the plasma. We will continue these analytical and simulation studies with particular emphasis on developing improved theoretical models with predictive capability for determining the degree of charge and current neutralization over a wide range of system parameters, including transient beampulse conditions. These studies will include detailed comparison with LSP simulations and other simulation codes, as well as available experimental results.

Simulation studies of final pulse compression: Strong final pulse compression will be a key element of the Integrated Beam Experiments (IBX). A Ph.D. thesis exploring this area was recently completed. As part of this work, the HERMES model [23, 24] was developed within the WARP code framework [9, 25]. This model solves the transverse envelope equations for a set of beam "slices" which are mutually coupled via a longitudinal Lagrangian fluid model. The longitudinal field is obtained either by WARP's $(r, z)$ field solver, or by a Bessel function

expansion. Several drift compression systems were designed within the constraints of the preservation of a "matched" beam and effective velocity "tilt" removal by space charge at the end of compression, based on a given desired pulse shape at the end of drift compression. The occurrence of mismatches due to a rapidly increasing current was analyzed. In addition, the sensitivity of drift compression to errors in the initial velocity tilt and current profile was studied.

\section{STUDIES SUPPORTING DRIVER-RELATED EXPERIMENTS}

\section{A. High Current Experiment (HCX)}

Extensive 3D and 2D WARP PIC simulations of the injector and matching section of the HCX [27] have been carried out to understand the phase space structure of the beam emerging from the injector and to guide improvements. Simulations and theory have also been employed to explore the consequences of collective modes launched by phase space distortions of an imperfect injector. It was determined that the relaxation of such modes should not result in unacceptably high emittance growth [21]. Matching and envelope analyses have been carried out in support of lattice retrofits and error analyses, and simulation data have been employed to support the choice in aperture of the electrostatic quadrupoles now in production. Continuing $2 \mathrm{D}$ and $3 \mathrm{D}$ simulations are being carried out to support 
the near-term experimental program by determining the expected beam properties, aiding in machine tuning and diagnostic interpretation, suggesting additional diagnostics and measurement locations, and guiding continued improvements in the injector and matching section [28].

Studies have been carried out for the HCX lattice extended with 20-30 additional electric and/or magnetic quadrupoles. Syncopated FODO lattices were developed consistent with magnet technology in development and the full range of experimental needs. It was found that a small amount of syncopation led to negligible degradation in current transport and allowed axial space in the low energy beam lattice for cryostat terminations, diagnostics, etc. A large series of detailed WARP simulations were carried out [28] to parametrically explore the influence of nonlinear magnetic field errors, image charges, beam mismatch induced by random and systematic errors in quadrupole excitation, halo, beam control and steering, skew couplings from rotational misalignments, and the effects of collective modes launched from systematic and random phase space distortions. These studies are being used to establish design tolerances, etc., to prevent particle loss.

Simulation results predict that the dynamic aperture in the electrostatic focusing transport section will be set by particle loss [29]. These studies use both idealized initial distribution functions (semi-Gaussian) and initial distribution functions calculated from first principles as transported from the source (including a 3D WARP PIC calculation in the injector). Most recently, we have begun carrying out studies wherein the simulated beam was initialized part-way through the experiment, using a transverse 4-D beam particle distribution $f\left(x, y, p_{x}, p_{y}\right)$ synthesized from experimental slit-scan measurements. This procedure has been explored using a simulated beam from WARP, whereby the true transverse 4-D distribution is known, as well as using preliminary experimental data. This work is helping to establish requirements for the experimental diagnostics; in particular, it has made clear the value of a crossed-slit spatial density scan, in addition to the more common parallel-slit emittance scans $[30,31]$.

Here we present an example of an "integrated" calculation. This type of calculation will be important for studies of employed for exploration and planning of future experiments, such as an IBX or IRE. The calculation begins by using WARP in its 3D time-dependent mode on a $64 \times 64 \times 640$ grid, computing space-charge limited emission from the source as the triode voltages ramp up. This first "run" includes all of the electrostatic quadrupole (ESQ) 
injector structure, as shown in Fig. 1(a), a frame from a movie of this calculation. The beam head is "mismatched" transversely (leading to less than $0.1 \%$ particle loss) because its energy differs from the nominal, a result of the overly-slow 800 ns full-rise time of the gate voltage. A 400-ns full-rise time leads to no loss (the actual rise time in the experiment is slightly less than $800 \mathrm{~ns}$ ). Then, because of the desirability of efficiency and high grid resolution $(512 \times 512)$ in the much thinner downstream beam line, the particle data at the exit plane of the ESQ is saved over the flat-top of the pulse, and used to initiate a WARPxy "slice" calculation (in a steady-flow approximation with a 4-D transverse phase space) of the mid-pulse behavior through the matching section and ten electrostatic quadrupole lenses of the HCX transport line. A view of this later stage of the calculation is shown in Fig. 1(b).

Ongoing research is exploring the consequences of lost particles in both electric and magnetic focusing channels, in regimes relevant to both near-term experiments on HCX and to fusion drivers. Studies of the sources and sinks of stray electrons in a heavy ion accelerator, their dynamical lifetimes in the presence of the beams' space-charge field as well as the applied magnetic focusing and induction acceleration fields, and the consequences of their presence at various levels, are receiving enhanced emphasis. Collaborations on related loss issues in accelerators for other applications have been established. These studies are using analysis, as well as PIC simulations [32].

\section{B. Injector experiments}

Accurate simulation of injector systems remains a challenging area, because of the need to avoid introducing any numerical artifacts into the beam distribution. Here two approaches are being pursued. One approach involves a large-diameter hot-plate source followed by an ESQ focusing and accelerating column, as currently employed in the HCX experiments. Extensive simulations of this system are being carried out using the WARP particle-incell code, as described above. Studies of the source region in detail are also underway [33]. Another approach involves the merging of a large number ( 100) of micro-beamlets,

each with high current density, offering the promise of a compact system [34]. However, the approach also poses physics and engineering challenges, as well as the computational challenge of a wide range of scales [35]. We are simulating all aspects of this approach. This work includes studies of the propagation of the beamlets from their individual sources 


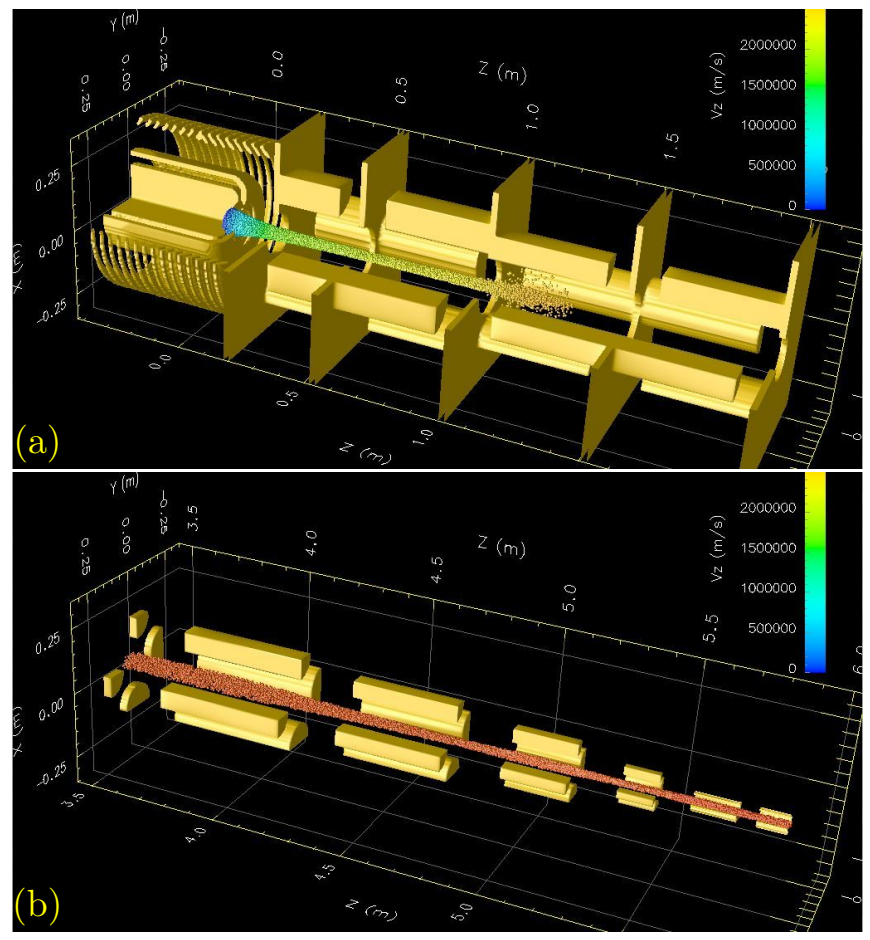

FIG. 1: (Color) Depictions of (a) the beam head passing through the HCX injector, and (b) the beam in the HCX matching section and entrance to the transport line.

through their individual aperture columns (as single beamlets, to check for aberrations; and as a coupled array of beamlets, to check for inter-beam forces and deflections, which appear to be very small). It also includes studies of the merging process, beginning at the point where the beamlets begin to "see" each other. Here we present an example of a merging calculation. In this run (part of a parameter study), 91 semi-Gaussian beamlets (each 0.006 A, $0.003 \pi-\mathrm{mm}-\mathrm{mr}$ ) exit their aperture columns at $1.2 \mathrm{MeV}$ and are accelerated across a gap to $1.6 \mathrm{MeV}$, where they enter a common transport line. The 2D WARP simulation shown here employed 29 M particles, 1024×1024 grid, 4000 steps, using 18.2 hours on 64 IBM SP processors. Figure 2 shows selected frames from a computer-generated movie. The resulting beam has comparable emittance to that observed in simulations of the ESQ injector for HCX, but differs qualitatively in that the phase space has been "diluted" by the entrainment of empty volume, rather than "distorted" through focusing aberrations. 

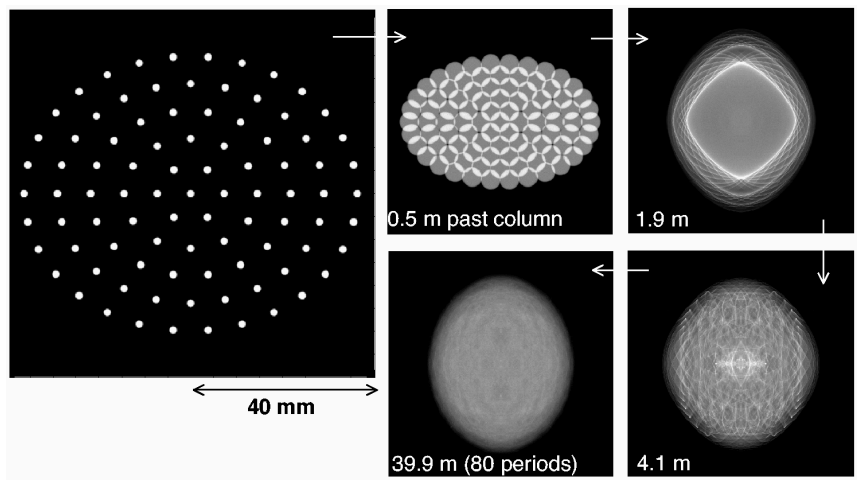

FIG. 2: A sequence of "snapshots" of the merging beamlets, as computed using a high-resolution WARPxy simulation.

\section{STUDIES OF BEAM PROPAGATION IN FUSION-CHAMBER PLASMAS}

Ion beams must be transported and focused over several meters through the fusion chamber to the target [36]. This "standoff" distance prevents damage to the final focus section of the accelerator from the target explosion [37]. Ballistic transport uses a final focusing lens just outside the chamber and a supply of electrons to provide neutralization (neutralized ballistic transport) [38]; the detailed physics of the neutralization process is one of the key areas to be addressed by the NTX experiments [39, 40], along with the optics of final focusing [41]. Pinched transport uses a final lens to focus each beam to a small radius at the entrance to the chamber, and then the beam propagates at small radius to the target [42]. All pinch modes depend on an azimuthal magnetic field $\left(B_{\theta}\right)$ to confine the beams. Assisted-pinched transport uses a preformed 50-kA channel, created in a gas (1-10 Torr) by a laser and a discharge electrical circuit, to create a frozen magnetic field before the heavy ion beam is injected [43-45]. Self-pinched transport uses the ion beam itself to break down a low-pressure gas (1-100 mTorr) [13, 46, 47], and the net self-magnetic field affords confinement. These transport schemes impact the designs of both the accelerator and the fusion target.

Simulation of these transport modes involves plasma densities that range from very weak to $>10^{16} \mathrm{~cm}^{-3}$. Neutralizing plasma is either created externally before the ion beam arrives, or is created by means of beam impact ionization and photoionization from the heated target. While standard particle-in-cell (PIC) codes are adequate for weak plasma conditions, a hybrid code that is capable of accurately modeling the dense plasma is necessary for electron 
densities above approximately $10^{13} \mathrm{~cm}^{-3}$. Above this density with a $\mathrm{keV}$ temperature, the Debye length becomes prohibitively small, necessitating cell sizes below 10 microns and time steps below 10 ps. Given a simulation distance of 3-6 meters, standard PIC simulations are problematic. Thus, use is made of the hybrid simulation codes LSP [48] and IPROP [49] that include detailed modeling of the gas breakdown from near vacuum to 5-Torr ambient gas pressure. LSP is used in the weakly-collisional regimes of neutralized-ballistic transport [50] and self-pinched transport $[13,46]$, whereas IPROP is used for the more collisional assisted-pinched transport regime [51].

A hybrid algorithm has been developed and implemented in the 3-D parallel PIC code LSP. An energy-conserving[52] implicit algorithm including particle collisions and gas breakdown models has been added with both electrostatic and electromagnetic field solvers. This treatment relaxes the usual limitations on time step and cell sizes associated with the cyclotron and plasma frequencies and the Debye length. A recent improvement in the code involves an implementation of a cloud-in-cell (CIC) treatment [53]. The treatment greatly reduces particle noise inherent in an energy-conserving algorithm. The LSP code also has algorithms to model charged particle and neutral particle collisions. To avoid problems associated with numerical cooling of highly implicit kinetic electrons, we include an implicit PIC fluid model for the electrons. The equation of motion for a fluid electron parcel is identical to that of a kinetic particle, except for scattering terms. A pressure-gradient force term, to model electron-electron collisions, and a frictional force between the electron and other species replace elastic scattering events for kinetic particles.

LSP has been used recently to make "realistic" simulations of driver-like "foot" and main pulses in a target chamber. In these calculations, the beam enters the chamber through a conical three-meter beam port with layers of hydrogen plasma near each end to preneutralize the beam, then drifts three more meters through low-density $\mathrm{BeF}_{2}$ to a 5 -mm radius target. Beam parameters are close to values used in ongoing optimzation studies, so main pulses initially have $2.8 \mathrm{kA}$ of $2.5 \mathrm{GeV} \mathrm{Xe}^{+1}$ ions, and foot pulses have $947 \mathrm{~A}$ of 1.9 GeV ions. These runs allow Child-Langmuir electron emission from conducting walls, and several also include time-dependent photo-stripping of the beam and photoionization of the background gas by X rays from the heated target [54]. Although these photoionization processes have major effects on the beam charge state and on the density of free electrons near the target, their overall effect in the cases examined to date is a modest reduction in 
the beam spot size [55]. Results from a typical main-pulse simulation are shown in Fig. 3. Here, the beam density and electron densities from several sources are shown after 80 ns of transport. At this time, electrons from the plasma layers and from the beam-pipe walls provide the largest share of beam neutralization, although neutralization by photoionization electrons becomes dominant as the beam reaches the target. An important finding of this and similar simulations is that the beam waist for either type of pulse is close to values required by current distributed-radiator targets [56].
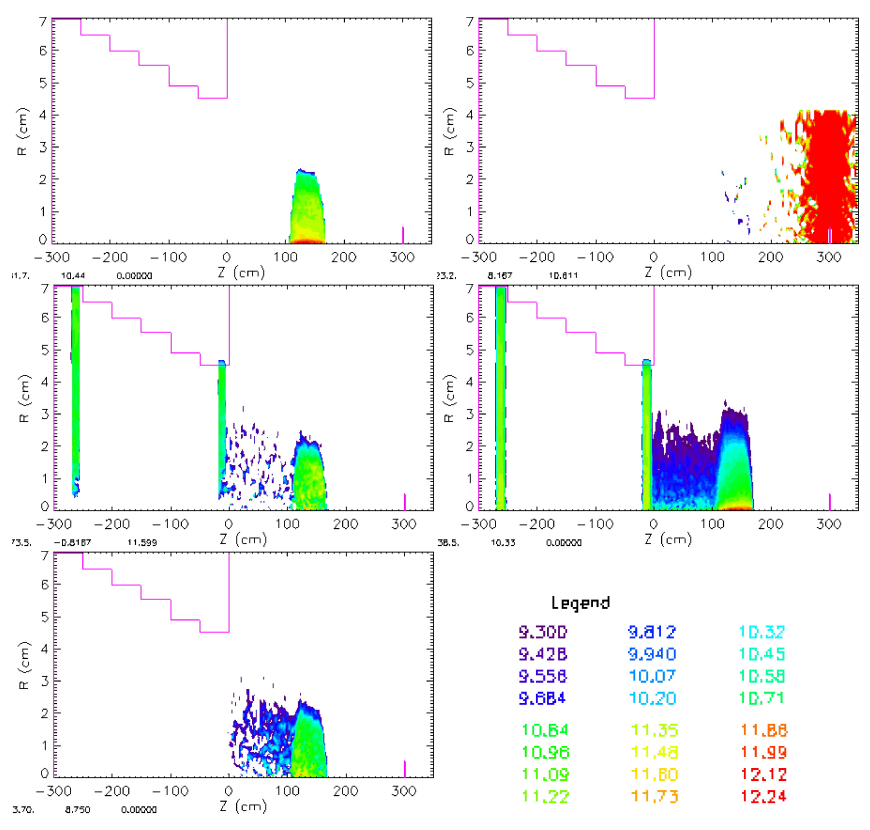

FIG. 3: (Color) The logarithm of the density of the (a) $\mathrm{Xe}^{+}$ions, (b) photoionization electrons, (c) plasma electrons, (d) wall emission electrons, and (e) impact ionization electrons, all at $80 \mathrm{~ns}$ into the LSP chamber transport simulation.

The IPROP code is presently being used to study, in 2-D and 3-D, the effects of beam self-fields and gas interaction in assisted-pinched transport. Such runs are particularly challenging because the ion beams are combined in two, each with mega-ampere electrical currents, and are highly stripped $(Z>60)$. The IPROP simulations are fully electromagnetic and make use of a tensor conductivity model. Fields and currents are decomposed in an arbitrary number of Fourier modes in the azimuthal direction. This decomposition allows for efficient simulation of 3-D resistive instabilities, such as the hose and filamentation instabilities, with only 2 modes. Thus, the simulation speed is close to that of a $2-\mathrm{D}$ simulation. The IPROP code treats the beam ions kinetically; however, the plasma is described with a 
two-fluid model. The plasma electron current density is determined from an Ohm's law that includes pressure and friction, as well as electromagnetic terms. The electron conductivity, $\sigma=c n_{e} / m_{e} \nu_{m}$, where $n_{e}$ is the electron density and $\nu_{m}$ is the momentum transfer frequency is calculated from Spitzer collisions. Electron densities are advanced using the continuity equation taking into account beam ionization that is so intense that multiple stripping by the beam must be modeled.

Recent IPROP simulations [51] wherein the beam is guided by the magnetic fields from a $50 \mathrm{kA}$ discharge channel have suggested that up to $90 \%$ of the beam energy reaches the target. A significant increase in the net current above the $50 \mathrm{kA}$ level degrades transport due to inductive losses and halo formation. The calculations show that the beam heats the gas sufficiently quickly to yield a high conductivity that resists the growth in self-magnetic fields to tolerable levels. Furthermore, 3-D simulations predict that the resistive hose instability will not disrupt the beam propagation.

\section{FUTURE EXPERIMENTS AND SIMULATION CAPABILITIES}

We have begun developing the concept for the next major step in the development of a heavy ion fusion driver, the so-called Integrated Beam Experiment (IBX) (cf. [57]). The purpose of IBX is to explore in an integrated manner the processes and manipulations necessary for a heavy ion fusion induction accelerator. The experiments will demonstrate injection, acceleration, compression, bending and final focus of a heavy ion beam at significant line charge density. A primary scientific goal will be exploration of the longitudinal physics issues associated with compression of the bunch length by a factor of ten or more, while maintaining high beam quality (both transverse and longitudinal) and envelope and centroid control. Two preliminary conceptual designs (with flexible experimental options), a short pulse version (having a $250 \mathrm{~ns}$ initial pulse) and a longer pulse version (with a $2 \mu$ s initial pulse), have been outlined. The short-pulse design requires significantly smaller induction cores and a shorter drift compression length, with a resultant cost savings. The injector for the short-pulse design, however, requires further research to ensure that transients do not dominate the current pulse. The longer pulse design requires no extrapolation from present technology.

We developed an improved analytic model for the beam spot size on the fusion target 
based on a non-linear moment model, incorporating improvements in our understanding of chromatic effects, and allowing for the possibility of elliptical spots. We also recently adapted a model which includes emittance growth in the chamber [58] and self-pinching in the chamber $[12,59,60]$, and which captures some of the understanding gleaned from particle simulations and analysis of chamber propagation [40, 55]. These models have been incorporated into the systems code IBEAM, thereby improving the agreement between systems models and particle simulations.

Advanced code development is in progress in a number of areas. One key project is centering on the use of adaptive mesh refinement (AMR) in WARP, so that the mesh resolution is concentrated in those spatial regions where fine details of the field must be resolved. A prototype axisymmetric model using AMR is already available in WARP, and a full 3D capability, involving the merging of WARP with the existing CHOMBO package (itself being modified to accommodate particle simulation needs), is under development [61].

Other directions to be pursued for advanced algorithms include the generalization to alternating-gradient focusing of Vlasov methods which follow the evolution of the beam's phase-space density on a multidimensional grid [62], development of true multi-beam capabilities in both WARP and LSP, and implementation of novel magneto-inductive (Darwin) models for beam simulation into the full suite of HIF codes.

A key programmatic goal is the development of an integrated and detailed "source-totarget" HIF beam simulation capability. Testing and validating this integrated modeling capability will be a main objective of the IBX. In our proposed scenario for integrated modeling (Fig. 4), the beam is simulated from the source through the final focusing optic using WARP3d, and the particle and field data are then transferred into LSP where the simulation is carried through the chamber plasma environment to the fusion target. At that point the particle data is used to generate "ray" information for the ion beam source in the radiation-hydrodynamics target design code. To ensure fidelity, coupling of this consistent sequence of simulations with other, more detailed calculations of instabilities and other processes (analogous to those described herein, using BEST) will be important. Similarly, to understand beam halos quantitatively, the orbit-following capabilities of BEST, WARP with an extra-large number of particles, and/or extensions of the semi-Lagrangian Vlasov solver SLV may be employed in calculations over limited sections of the machine. 


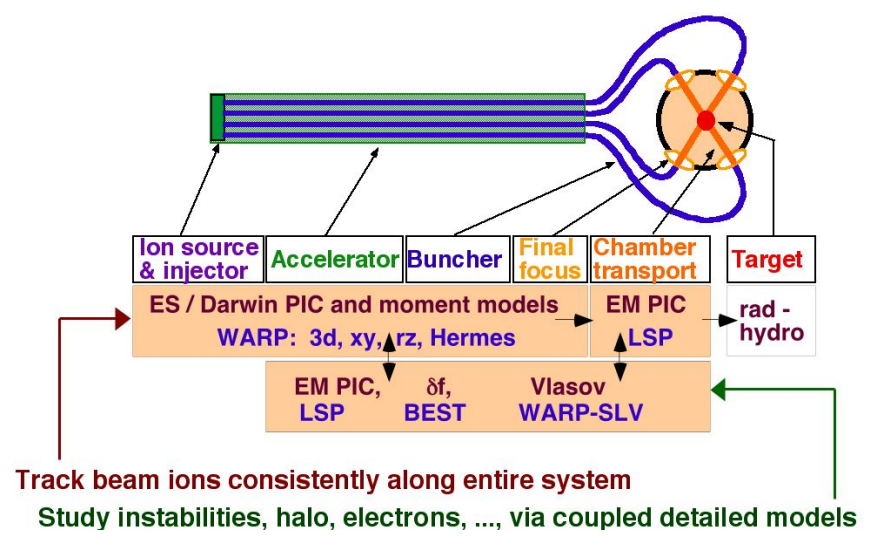

FIG. 4: (Color) A depiction of the strategy being pursued for consistent, detailed end-to-end beam simulation.

\section{Acknowledgments}

This work was performed under the auspices of the U.S. Department of Energy by the University of California, Lawrence Livermore and Lawrence Berkeley National Laboratories under Contract Nos. W-7405-Eng-48 and DE-AC03-76SF00098, and by the Princeton Plasma Physics Laboratory under Contract No. DE-AC02-76CH03073.

[1] Nuclear Instruments and Methods in Physics Research (2001), 464A, pp. 1-674, and references therein.

[2] Davidson, R. C., and Qin, H. (2001), Physics of Intense Charged Particle Beams in High Energy Accelerators (World Scientific, Singapore).

[3] Qin, H., Davidson, R. C., Lee, W. W., and Startsev, E. (2001), Proceedings of the 2001 Particle Accelerator Conference, pp. 696-701.

[4] Qin, H., Davidson, R. C., and Lee, W. W. (2000), Physical Review Special Topics on Accelerators and Beams 3, 084401; 109901.

[5] Uhm, H. S., Davidson, R. C., and Kaganovich, I. (2001), Phys. Plasmas 8, 4637.

[6] Davidson, R. C., and Qin, H. (2000), Physics Letters A270, 170.

[7] Davidson, R. C., Qin, H., Stoltz, P., and Wang, T. -S. (1999), Physical Review Special Topics on Accelerators and Beams 2, 054401.

[8] Startsev, E. A., Davidson, R. C., and Qin, H. (2002), Phys. Plasmas 9, in press (2002). 
[9] Friedman, A. Grote, D. P., and Haber, I. (1992), Phys. Fluids B4, 2203.

[10] Friedman, A., Bangerter, R. O., Callahan, D. A., Grote, D. P., Langdon, A. B., and Haber, I., Proc. 2nd Euro. Part. Accel. Conf., Nice, June 12-16, 1990, pp. 1699-1701 (Editions Frontieres, 1990).

[11] Harris, E. G. (1959), Phys. Rev. Lett. 2, 34.

[12] Kaganovich, I., Shvets, G., Startsev, E., and Davidson, R. C. (2001), Phys. Plasmas 8, 4180.

[13] Rose, D. V., Ottinger, P. F., Welch, D. R., Oliver, B. V., and Olson, S. L. (1999), Phys. Plasmas 6, 4094.

[14] Ottinger, P. F., et al. (2000), Phys. Plasmas 7, 346; Chen, P. (1987), Particle Accel. 20, 171.

[15] Hahn, K., and Lee, E. P. (1996), J. Fusion Engineering and Design 32, 417.

[16] Qin, H., and Davidson, R. C. (2002), Physical Review Special Topics on Accelerators and Beams 5, 034401.

[17] Davidson, R. C., and Qin, H. (2002), Physical Review Special Topics on Accelerators and Beams 4, 104401.

[18] Tzenov, S. T., and Davidson, R. C. (2002), Physical Review Special Topics on Accelerators and Beams 5, 021001.

[19] Davidson, R. C., Qin, H., Lee, W. W., and Startsev, E. (2002), Phys. Plasmas 9, 340.

[20] Davidson, R. C. (1998), Phys. Rev. Lett. 81, 991.

[21] Lund, S. M., Barnard, J. J., Davidson, R. C., Lee, E. P., and Qin, H., (2002), submitted for publication.

[22] Lee, E. P., "Precision solution of the coupled beam envelope equations for a periodic quadrupole lattice with space charge," Submitted to Physics of Plasmas, 2002.

[23] de Hoon, M. J. L., "Drift Compression and Final Focus Systems for Heavy Ion Inertial Fusion," Ph.D. thesis, University of California, Berkeley, 2001.

[24] de Hoon, M. J. L., Lee, E. P., Barnard, J. J., and Friedman, A., "Cold phase fluid model of the longitudinal dynamics of space-charged dominated beams," Submitted to Physics of Plasmas, 2002; LBNL-49654.

[25] Grote, D. P., Friedman, A., Craig, G. D., Haber, I., and Sharp, W. M., Nucl. Instr. and Meth. in Phys. Res. A. 464, 563 (2001).

[26] Stoltz, P. H., Davidson, R. C., and Lee, W. W. (1999), Phys. Plasmas 6, 298.

[27] Seidl, P. A., et. al., these Proceedings. 
[28] Lund, S. M., et. al., these Proceedings.

[29] Celata, C.M., et. al., these Proceedings.

[30] Friedman, A., et. al., these Proceedings.

[31] Friedman, A., Grote, D. P., Celata, C. M., and Staples, J. W., "Synthesizing a fourdimensional beam particle distribution from multiple two-dimensional views," submitted to Phys. Rev. Special Topics - Accel. and Beams, 2002.

[32] Molvik, A. W., et. al., these Proceedings.

[33] Haber, I., these Proceedings.

[34] Kwan, J. W., et. al., these Proceedings.

[35] Grote, D. P. , et. al., "Design and Simulation of the Multi-Beamlet Injector for a High Current Accelerator," to be submitted to Phys. Rev. Special Topics - Accel. and Beams, 2002.

[36] Bangerter, R. O. (1998), Nucl. Instr. and Meth. Phys. Res. A 415, 3.

[37] Olson, C. L. (1982), J. Fusion Energy 1, 309.

[38] Logan, B. G., and Callahan, D. A. (1998), Nucl. Instr. and Meth. Phys. Res. A 415, 468.

[39] Olson, C. L., et. al., these Proceedings.

[40] Welch, D. R., et. al., these Proceedings.

[41] Henestroza, E., et. al., these Proceedings.

[42] Olson, C. L. (2001), Nucl. Instr. and Meth. Phys. Res. A 464, 118.

[43] Yu, S., et al. (1998), Nucl. Instr. and Meth. Phys. Res. A 415, 174.

[44] Olsen, J. N., and Leeper, R. J. (1982), J. Appl. Phys. 53, 3397.

[45] Ozaki, T., Miyamota, S., Imasaki, K., Nakan, S., and Yamanaka, C. (1985), J. Appl. Phys. $\mathbf{5 8}, 2145$.

[46] Welch, D. R., and Olson, C. L. (1996), J. Fusion Engineering and Design 32-33, 477.

[47] Ottinger, P. F., Rose, D. V., and Oliver, B. V. (1999), Phys. Plasmas 6, 3717.

[48] Hughes, T. P., Yu, S. S., and Clark, R. E. (1999), Phys. Rev. ST-AB 2, 110401; Welch, D. R., Rose, D. V., Oliver, B. V., and Clark, R. E. (2001), Nucl. Instr. and Meth. Phys. Res. A 464, 134 .

[49] Welch, D. R., Olson, C. L., and Sanford, T. W. L. (1994), Phys. Plasmas 1, 764.

[50] Rose, D. V., Welch, D. R., Oliver, B. V., Clark, R. E., Clark, W. M., and Friedman, A. (2001), Nucl. Instr. and Meth. Phys. Res. A 464, 299.

[51] Welch, D. R., Rose, D. V., Oliver, B. V., Genoni, T. C., Clark, R. E., Olson, C. L., and Yu, 
S. S. (2002), Phys. Plasmas, to be published.

[52] Birdsall, C. K., and Langdon, A. B. (1991), Plasma Physics Via Computer Simulation (Adam Hilger, Bristol, Philadelphia and New York, 1991), Chapter 10.

[53] Birdsall, C. K., and Fuss, D. (1997), J. Comp. Phys. 135, 141.

[54] Sharp, W. M., Callahan-Miller, D. A., Langdon, A. B., Armel, M. S., and Vay, J. -L. (2001), Nucl. Instr. and Meth. Phys. Res. 464, 284.

[55] Sharp, W. M., et. al., these Proceedings.

[56] Callahan-Miller, D. A., and Tabak, M. (1999), Nucl. Fusion 39, 883.

[57] Barnard, J. J., et. al., these Proceedings.

[58] Lee, E. P., Yu, S. S., and Barletta, W. A., "Phase Space Distortion of a Heavy Ion Beam Propagating Through a Reaction Vessel," Nuclear Fusion, 21, 961 (1981).

[59] Hahn, K. and Lee, E. P., "A study of stripped pinched beam transport for heavy ion fusion," Fusion Engineering and Design, 32-33, 417 (1996).

[60] Oliver, B. V., Genoni, T., Rose, D. V., and Welch, D. R., "Laminar equilibria of ion-beam neutralizing electrons in vacuum and plasma," presentation at Self-Pinch Workshop for Final Transport of Heavy Ion Beams, Danville, CA (2001).

[61] Vay, J.-L., et. al., these Proceedings.

[62] Sonnendrucker, E., Barnard, J. J., Friedman, A., Grote, D. P., and Lund, S. M., Nucl. Inst. and Meth. Phys. Res. A 464, 653-661 (2001). 